\title{
Phases and Transitions in Phantom Nematic Elastomer Membranes
}

\author{
Xiangjun Xing \\ Department of Physics, University of Illinois, Urbana-Champaign, IL 61801 \\ Leo Radzihovsky \\ Department of Physics, University of Colorado, Boulder, CO 80309
}

(Dated: May 9, 2018)

\begin{abstract}
Motivated by recently discovered unusual properties of bulk nematic elastomers, we study a phase diagram of liquid-crystalline polymerized phantom membranes, focusing on in-plane nematic order. We predict that such membranes should generically exhibit five phases, distinguished by their conformational and in-plane orientational properties, namely isotropic-crumpled, nematic-crumpled, isotropic-flat, nematic-flat and nematic-tubule phases. In the nematic-tubule phase, the membrane is extended along the direction of spontaneous nematic order and is crumpled in the other. The associated spontaneous symmetries breaking guarantees that the nematic-tubule is characterized by a conformational-orientational soft (Goldstone) mode and the concomitant vanishing of the in-plane shear modulus. We show that long-range orientational order of the nematic-tubule is maintained even in the presence of harmonic thermal fluctuations. However, it is likely that tubule's elastic properties are qualitatively modified by these fluctuations, that can be studied using a nonlinear elastic theory for the nematic tubule phase that we derive at the end of this paper.

PACS numbers: PACS:61.41.+e, 64.60.Fr, 64.60.Ak, 46.70.Hg
\end{abstract}

\section{INTRODUCTION}

Fluctuating polymerized [1] membranes [2] have attracted considerable interests in the past two decades. Probably the most striking property that distinguishes them from their one-dimensional polymer analogue and most other two-dimensional systems, is that polymerized membranes admit a low-temperature flat phase [3, 4, 5] characterized by a long-range order in membrane's normal. This phase is separated from a high-temperature crumpled phase, with randomly-oriented normals, by a thermodynamically-sharp transition [6, 7, 8] . At low temperatures, strong interplay between thermal fluctuations and elastic nonlinearities infinitely enhances the effective bending rigidity, which in turn stabilizes the flat phase against these very fluctuations $[3,4,[8,[9]$. The resulting flat phase exhibits rather unusual length-scale dependent elasticity, nonlinear response to external stress and a universal negative Poisson ratio. 4, 5]

More recently, stimulated by a number of possible anisotropic realizations, the role of elastic membrane anisotropy was considered by Radzihovsky and Toner 10. It was discovered that while permanent inplane anisotropy is unimportant at long scales within the crumpled and flat phases, it qualitatively modifies the global phase diagram of anisotropic polymerized membranes, leading to an intermediate (in its properties and location in the phase diagram) "tubule" phase. In the tubule phase the membrane is extended in one direction and crumpled in the other, with its crumpled boundary exhibiting statistics of a self-avoiding polymer. The usual crumpled-to-flat transition of an isotropic membrane is therefore generically split into two consecutive ones: crumpled-to-tubule and tubule-flat transitions. Fluctuations, nonlinear elasticity, and phase transitions into and out of the tubule phase have been studied extensively 10, with predictions dramatically confirmed by large-scale Monte Carlo simulations [11].

Motivated by unusual properties of nematic elastomers that have been recently discovered 12, 13, 14, 15, 16, 17, 18], i.e. a spontaneous nematic order in an amorphous solid phase and resulting soft elasticity characterized by one vanishing shear modulus, we have previously studied fluctuations and elasticity of a flat tethered membrane that exhibits spontaneous in-plane anisotropy, i.e. a nematic order 19]. Such spontaneous order guarantees the existence of a zero-energy in-plane deformation mode corresponding to a simultaneous reorientation of nematic and uniaxial distortion axes, and hence vanishing of an in-plane shear modulus. As in other "soft" fluctuating systems [20], we found that elastic nonlinearities become qualitatively important in determining membrane's longscale elasticity and conformations. However, due to the vanishing of an in-plane shear modulus, the elasticity of flat nematic elastomer membranes differ qualitatively from that of isotropic and that of explicitly anisotropic membranes: a renormalization analysis of the undulation nonlinearity shows that nematic-flat phase is characterized by a conventional, length scale independent bendingrigidity modulus. 19, 21.

While bulk nematic elastomers and gels have received considerable experimental and theoretical attention, properties of equally interesting two-dimensional system, i.e. nematic elastomer membranes, have, to the best of our knowledge have not been explored in any detail. A few (rather idealized) experimental realizations of such spontaneously anisotropic nematic membranes can be envisioned. For example, one may prepare a nematic elastomer sheet in its isotropic phase and lower the temperature of the elastomer membrane into its ne- 
matic state. The chemical structure of liquid crystalline mesogenic units in such nematic elastomer should be chosen such that they prefer alignment parallel to the membrane surface. Liquid-crystalline polymers adsorbed onto a polymerized membrane can also develop a spontaneous nematic order at sufficiently high density 22], which in turn will act as a spontaneous elastic anisotropy to the membrane elasticity. The elastic properties of lipid bilayers with spontaneous tilt order should be almost identical to nematic elastomer membranes that we study in this paper 23]. Finally, other types of liquid crystalline orders may also be realized in polymerized membranes. For example, two dimensional smectic order may spontaneously develops when temperature is further lowered in a nematically-ordered elastomer membrane. This can also be taken into account using a straightforward generalization of our model.

In this paper we continue to explore the physics of phantom 24] liquid-crystalline tethered membranes, focusing on the overall phase diagram that results from the interplay of membrane's in-plane nematic and conformational orders. As discussed in detail in Sec. IIII we find that in the isotropic sector of the phase diagram, characterized by a vanishing in-plane nematic order, the system is identical to the usual isotropic tethered membranes, and therefore exhibits well studied isotropic-crumpled (IC) and isotropic-flat (IF) phases. Development of nematic order generically leads to three additional phases: the nematic-crumpled (NC), nematic-tubule (NT) and nematic-flat (NF) phases. Different phases can be distinguished by different expectation values of the nematic order parameter and the metric tensor. These results are summarized by a mean-field global phase-diagram in Fig. [1]

We also study harmonic fluctuations within the most interesting nematic-tubule phase. As we discuss in Sec. [V] and show schematically in Fig. 2] the geometry and fluctuations of a nematic tubule, with an intrinsic size $L \times L$, are characterized by its average thickness $R_{G}(L)$ and root-mean fluctuations $h_{r m s}(L)$ about its energetically preferred extended state, that scale as:

$$
R_{G}(L) \propto L^{\nu}, \quad h_{\mathrm{rms}}(L) \propto L^{\theta},
$$

where $\nu$ and $\theta$ are universal exponents. 10. For idealized "phantom" membranes $\nu=1 / 4$ and $\theta=1$, but are expected to be significantly modified by the self-avoidance and elastic nonlinearities. 10 These conformational properties (or the absence thereof) can also be used as indicators of phase transitions into/out-of the nematic tubule phase, accompanied by usual thermodynamic singularities, e.g., in the heat-capacity. For a more detailed discussion of the scaling properties of polymerized tubules and transitions into/out-of the tubule phase, we refer the reader to Ref 10 .

We also derive a fully rotationally invariant nonlinear elasticity characterizing nematic tubules. We find that because of the spontaneous nature of the in-plane nematic order, the nonlinear elastic free energy of the ne-

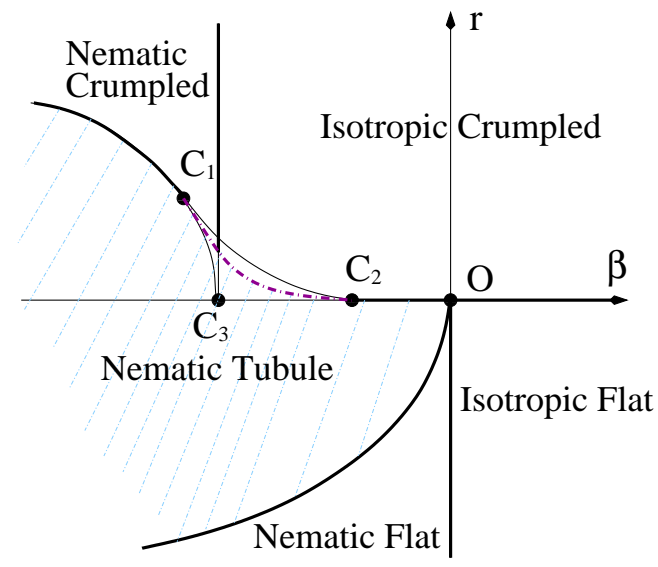

FIG. 1: (Schematic mean-field phase diagram for a polymerized membrane with spontaneous in-plane nematic order. Parameters $r$ and $\beta$ drive crumpling transition and isotropicnematic transition respectively and are defined in Sec II and Sec. III Nematic-tubule phase is the stable phase in the shaded region. In the triangle region between three critical points $C_{1}, C_{2}$ and $C_{3}$, two phases are metastable. The dash line connecting $C_{1}$ and $C_{2}$ is a (schematic) first-order transition line between isotropic/nematic-crumpled phases and the nematic-tubule phase.

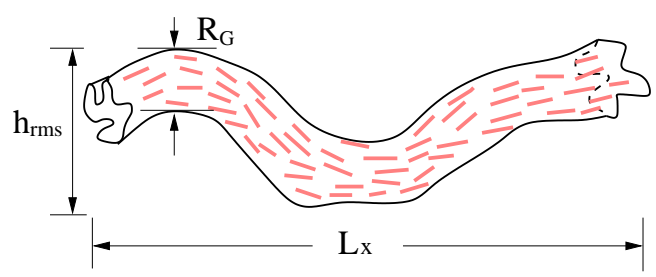

FIG. 2: Conformational properties of the nematic tubule phase. Average thickness $R_{G}$ and root-mean fluctuation $h \mathrm{rms}$ are defined in Eqs. (49). Small rods, aligned on the membrane's surface schematically indicate existence of the inplane nematic order.

matic tubule differs qualitatively from the tubule phase of a permanently anisotropic membrane [10]. We leave the full analysis of tubule's elastic nonlinearities and thermal fluctuations to a future research. Here, we will have little to say on the very interesting and important question of the effects of heterogeneity, associated with the random cross-linking of elastomers. Based on a recent body of work on conventional liquid crystals confined in random environments [25, 26] and randomly polymerized membranes [27], we expect that at sufficiently long scales, even weak heterogeneities will lead to qualitatively important modifications of some of the predictions made in this paper. Understanding these will be essential for a direct comparison with experiments [28]. We plan to explore these in the future.

The body of this paper is organized as follows. In Section [II we introduce a Landau model for a liquid- 
crystalline tethered membrane, whose mean field phase diagram we map out in Section [III In Section [IV] we focus on the most interesting phase, the nematic-tubule and study its thermal fluctuations at the harmonic level. In Section IVB we formally integrate out the nematic order parameter and derive an effective fully nonlinear and rotationally-invariant elastic free energy for the nematic tubule phase.

\section{MODEL OF NEMATIC ELASTOMER MEMBRANE}

Nematic elastomer membranes are direct generalization of permanently anisotropic tethered membranes studied in Ref. [10], with the in-plane elastic anisotropy chosen by a spontaneously, local nematic order. The latter plays the role of a new degree of freedom that fluctuates at a finite temperature. As for an ordinary tethered membrane, its geometric conformation (state) is described by a three-dimensional vector-field $\mathbf{R}(\mathbf{x})$, that specifies the position (embedding) of the referencespace mass point $\mathbf{x}$ in the three dimensional target space. Throughout this paper, we will use a lower-case bold-face characters, such as $\mathbf{x}=x_{a} \hat{e}_{a}=x \hat{x}+y \hat{y}$, to denote twodimensional vectors in the reference space and upper-case bold-face character such as $\mathbf{R}=X \hat{X}+Y \hat{Y}+Z \hat{Z}$ to denote three-dimensional vectors in the target space, respectively spanned by a set of mutually orthogonal unit vectors, $\left\{\hat{e}_{1}=\hat{x}, \hat{e}_{2}=\hat{y}\right\}$ and $\{\hat{X}, \hat{Y}, \hat{Z}\}$.

The elastic free energy density of the membrane can be conveniently described as a function of a reference-space metric tensor $\underline{\underline{g}}$

$$
g_{a b}=\frac{\partial \mathbf{R}}{\partial x_{a}} \cdot \frac{\partial \mathbf{R}}{\partial x_{b}},
$$

inherited from the Euclidean metric $\delta_{i j}$ of the embedding space. By construction, $g_{a b}$ is explicitly a targetspace scalar (i.e., rotationally invariant in the embedding space), rank-2 reference-space tensor, and is nonnegative. The thermal expectation value of the metric tensor, $\langle\underline{g}\rangle$ can be used to distinguish different phases of a tethered membrane. More specifically, two eigenvalues of $\langle\underline{\underline{g}}\rangle$ describe whether and how the membrane is extended along two directions in its reference space. For example, $\langle\underline{g}\rangle$ vanishes in the crumpled phase, and is proportional to the identity matrix in the flat phase, with the common eigenvalue describing the relative size of the flat membrane in the three dimensional target space. In the tubule phase of an intrinsically anisotropic membrane, $\langle\underline{\underline{g}}\rangle$ has only one positive eigenvalue, which corresponds to the fact that the membrane is extended along one direction and crumpled along others. Therefore $\langle\underline{\underline{g}}\rangle$ has all the properties of a physical order parameter.

Nematic elastomer membranes are in addition characterized by a local in-plane nematic order parameter field
$\underline{\underline{Q}}(\mathbf{x})$. Characterizing membrane's spontaneous elastic anisotropy, $\underline{\underline{Q}}$ describes an intrinsic property of the membrane and is a second-rank symmetric traceless tensor in the two dimensional reference space, with components $Q_{a b}, a, b=x, y$ and $\sum_{a} Q_{a a}=0$, and is a target-space scalar [29]. Landau free energy density for our model of a nematic elastomer membrane is a rotationally-invariant (both in the reference and target spaces) function of $\underline{\underline{g}}$, $\underline{\underline{Q}}$

$$
\tilde{f}[\underline{\underline{g}}, \underline{\underline{Q}}]=f_{e}[\underline{\underline{g}}]+f_{n}[\underline{\underline{Q}}]+f_{e n}[\underline{\underline{g}}, \underline{\underline{Q}}],
$$

where $f_{e}$ is the elastic free energy density for an isotropic polymerized membrane [7],

$$
f_{e}[\underline{\underline{g}}]=\frac{\kappa}{2}\left(\nabla^{2} \mathbf{R}\right)^{2}+r^{\prime} \operatorname{Tr} \underline{\underline{g}}+\frac{\lambda}{2}(\operatorname{Tr} \underline{\underline{g}})^{2}+\mu \operatorname{Tr} \underline{\underline{g}}^{2} .
$$

$f_{n}$ is the Landau-deGennes free energy density for a nematic-isotropic transition in two dimensions

$$
f_{n}=\frac{K}{2}(\nabla \underline{\underline{Q}})^{2}+\frac{\beta^{\prime}}{2} \operatorname{Tr} \underline{\underline{Q}}^{2}+\frac{v}{2}\left(\operatorname{Tr} \underline{\underline{Q^{2}}}\right)^{2} .
$$

It reflects the special feature of nematic-isotropic transition in two dimensions, namely, the absence of the usual cubic invariant $\operatorname{Tr} \underline{\underline{Q}}^{3}$ that vanishes identically. This allows nematic-isotropic transitions in two dimensions to be continuous [30].

The last part of $\tilde{f}$, Eq. 3 is the coupling between nematic order and the elastic degrees of freedom, which, to lowest order, is given by:

$$
f_{\text {en }}=-\alpha \operatorname{Tr} \underline{\underline{Q g}}=-\alpha \operatorname{Tr} \underline{\underline{Q \tilde{g}}},
$$

where $\underline{\underline{g}}=\underline{\underline{g}}-(\operatorname{Tr} \underline{\underline{g}}) \underline{\underline{I}} / 2$ is the traceless part of the metric tensor. In the presence of such tensorial nemato-elastic coupling, nematic order $Q$ acts as a uniaxial shear stress and therefore renders the in-plane elasticity anisotropic inside a nematic phase. Concomitantly, an elastic distortion characterized by an anisotropic metric tensor acts as a quadrupolar field inducing nematic order. As usual in Landau description, although other higher order couplings exist, such as for example $\gamma \operatorname{Tr} \underline{\underline{ }} \operatorname{Tr} \underline{\underline{Q}}{ }^{2}$, they do not lead to any new qualitative effects, and, furthermore, for weak nematic order, are quantitatively subdominant. Their primary effect is to slightly change the locus of phase boundaries without changing their topology. We will therefore ignore them in this paper.

We can also formally and perturbatively integrate out the nematic order parameter $\underline{Q}$ in Eq. (3) and obtain an effective elastic free energy density formulated purely in terms of the metric tensor. Up to fourth order in $\underline{\underline{g}}$, it is given by

$$
\begin{aligned}
\left.f_{\mathrm{eff}} \underline{\underline{g}}\right]= & \frac{1}{2} \kappa\left(\nabla^{2} \mathbf{r}\right)^{2}+\frac{1}{2} t \operatorname{Tr} \underline{\underline{g}}+u\left(\operatorname{Tr} \underline{\underline{\tilde{g}}}^{2}\right)+v(\operatorname{Tr} \underline{\underline{g}})^{2} \\
& +B\left(\operatorname{Tr} \underline{\underline{\tilde{g}}}^{2}\right)^{2}+C \operatorname{Tr} g \operatorname{Tr} \underline{\underline{\tilde{g}}}^{2},
\end{aligned}
$$


and agrees with the purely elastic model used in our previous study of the nematic-flat phase 19]. Although such effective model can also be used to study the phase diagram and other phases, it cannot distinguish the nematiccrumpled and the isotropic-crumpled phases, since both are characterized by a vanishing metric tensor. Therefore, depending on the equilibrium value of the metric tensor, controlled by model parameters, $f_{\text {eff }}$ predicts a crumpled phase, a spontaneous tubule phase (nematictubule phase), an isotropic-flat phase, and a spontaneous anisotropic flat phase (nematic-flat phase). Here, the usual crumpled-to-flat transition is determined by changing the sign of $t$, while the crumpled-to-tubule transition is driven by a sign of $u \propto \mu$. This purely elastic approach has the advantage of simplicity, stemming from absence of the nematic order parameter. In Sec IV we will use this effective elastic free energy to study the nonlinear elasticity in the nematic-tubule phase.

\section{MEAN-FIELD PHASE DIAGRAM}

An isotropic polymerized membrane with elastic free energy density, Eq. (4) undergoes a continuous 31] crumpling transition as $r^{\prime}$ is tuned from positive to negative []. On the other hand, $f_{n}$, Eq. (5) exhibits a second-order isotropic-nematic transition as $\beta^{\prime}$ changes sign [30]. When both $r^{\prime}$ and $\beta^{\prime}$ are small, a competition between these two types of order becomes important.

To work out the mean-field phase diagram, we minimize the free energy density Eq. (3) over the metric tensor $\underline{\underline{g}}$ and the nematic order parameter $\underline{\underline{Q}}$. To this end, it is convenient to rescale both order parameters $\underline{\underline{g}}$ and $\underline{\underline{Q}}$ as well as the total free energy Eq. 3] as following,

$$
\begin{aligned}
& \underline{\underline{Q}} \rightarrow \frac{\alpha}{4 \sqrt{\mu v}} \underline{\underline{Q}} \\
& \underline{\underline{g}} \rightarrow \frac{\alpha^{2}}{16 \sqrt{\mu^{3} v}} \underline{\underline{g}} \\
& \tilde{f} \rightarrow \frac{\alpha^{4}}{256 \mu^{2} v} f
\end{aligned}
$$

Furthermore, it is clear that the free energy is minimized by uniform nematic order and metric tensor. Therefore we can ignore the two higher derivative terms $\kappa, K$ appearing in Eqs. (4) and (5), respectively. The resulting rescaled total free energy density has the following simple form:

$$
\begin{aligned}
f \underline{\underline{\underline{g}}, \underline{Q}]} & =\frac{\epsilon}{2}(\operatorname{Tr}(\underline{\underline{g}}+r \underline{\underline{I}}-\underline{\underline{Q}}))^{2}+\operatorname{Tr}(\underline{\underline{g}}+r \underline{\underline{I}}-\underline{\underline{Q}})^{2} \\
& +\frac{1}{2}\left(\operatorname{Tr} \underline{\underline{Q}}^{2}+\beta\right)^{2}
\end{aligned}
$$

where

$$
\begin{aligned}
& r=\frac{8 r^{\prime} \sqrt{\mu^{3} v}}{\alpha^{2}(\lambda+\mu)}, \\
& \beta=\frac{8 \mu \beta^{\prime}}{\alpha^{2}}-1, \\
& \epsilon=\frac{\lambda}{\mu},
\end{aligned}
$$

and $\underline{\underline{I}}$ is the $2 \times 2$ identity matrix. Stability of the elastic free energy Eq. (4) requires $\mu>0$ and $\lambda+\mu>0$, therefore $\epsilon>-1$.

The linear coupling Eq. (6) between $\underline{\underline{g}}$ and $\underline{\underline{Q}}$ prefers same "orientation" (alignment of their eigenvectors) for these two tensors. Therefore in the ground state, where $\underline{\underline{g}}$ and $\underline{\underline{Q}}$ minimize the total free energy, Eq. (9), these two tensors commute. Using the coordinate system in which $\underline{g}$ and $\underline{Q}$ are simultaneously diagonal, they can be parameterized as:

$$
\underline{\underline{g}}=\left(\begin{array}{cc}
g_{1} & 0 \\
0 & g_{2}
\end{array}\right), \quad \underline{\underline{Q}}=\left(\begin{array}{cc}
S & 0 \\
0 & -S
\end{array}\right),
$$

where $g_{1}$ and $g_{2}$ are two non-negative eigenvalues of the metric tensor $\underline{\underline{g}}$, and $S$ a non-negative magnitude of the nematic order. Substituting these into free energy $f$, Eq. (9), we find that it can be written as

$$
f=\frac{1}{2} f_{g}\left[g_{1}, g_{2}, S\right]+\frac{1}{2} f_{S}[S]
$$

where

$$
\begin{aligned}
f_{g}\left[g_{1}, g_{2}, S\right] & =(2+\epsilon)\left(\left(g_{1}-\alpha_{1}\right)^{2}+\left(g_{2}-\alpha_{2}\right)^{2}\right) \\
& +2 \epsilon\left(g_{1}-\alpha_{1}\right)\left(g_{2}-\alpha_{2}\right) \\
& =(1+\epsilon)\left(g_{1}+g_{2}-\alpha_{1}-\alpha_{2}\right)^{2} \\
& +\left(g_{1}-g_{2}-\alpha_{1}+\alpha_{2}\right)^{2} \\
f_{S}[S] & =\left(S^{2}+\beta\right)^{2}
\end{aligned}
$$

and

$$
\begin{aligned}
& \alpha_{1}=(S-r), \\
& \alpha_{2}=-(S+r),
\end{aligned}
$$

with $\alpha_{1} \geq \alpha_{2}$, and $f_{g}, f_{S}$ non-negative definite.

To obtain a mean-field phase diagram, we need to minimize $f$, Eq. 12 over nonnegative $g_{1}, g_{2}$ and $S$ for given $r$ and $\beta$. Before doing that, however, it is useful to classify all possible phases according to ground state values of $g_{1}, g_{2}$ and $S$. It is easy to see that for vanishing nematic order, the free energy density, Eq. (3) reduces to that of an isotropic polymerized membrane, i.e. Eq. (4). An isotropic polymerized membrane is either in the isotropiccrumpled phase where $g_{1}=g_{2}=0$, or in the isotropic-flat phase, where $g_{1}=g_{2}>0$. In other words, a state with $g_{1} \neq g_{2}$ and $S=0$ cannot be a stable ground state. Conversely, anisotropy in the metric tensor, $g_{1} \neq g_{2}$ acts as 
an external field on the nematic order parameter $\underline{\underline{Q}}$, inevitably inducing finite nematic order. Therefore $\overline{S \text { must }}$ be finite whenever $g_{1} \neq g_{2}$. Similarly, a nonzero nematic order acts as an external shear stress tensor, which always induces a finite shear strain in a flat membrane (Hook's law), leading to elastic anisotropy with $g_{1} \neq g_{2}$. Therefore $g_{1}$ and $g_{2}$ cannot be identical in the presence of a finite nematic order, $S$. That is, if $S \neq 0$, the membrane can either be in a nematic-crumpled phase $\left(g_{1}=g_{2}=0\right)$, or a nematic-tubule phase $\left(g_{1}>g_{2}=0\right)$, or a nematicflat phase $\left(g_{1}>g_{2} \neq 0\right)$. We list the resulting five membrane phases in Table. [1

\begin{tabular}{|c|c|c|}
\hline \hline$g_{1}, g_{2}$ and $S$ & $S=0$ & $S \neq 0$ \\
\hline$g_{1}=g_{2}=0$ & Isotropic Crumpled & Nematic Crumpled \\
\hline$g_{1}=g_{2}>0$ & Isotropic Flat & Unstable \\
\hline$g_{1}>g_{2}>0$ & Unstable & Nematic Flat \\
\hline$g_{1}>g_{2}=0$ & Unstable & Nematic Tubule \\
\hline \hline
\end{tabular}

TABLE I: Possible phases of a nematic elastomer membrane.

We now minimize the rescaled free energy, Eq. (12) over $g_{1}, g_{2}$ and $S$. It is easy to see that if $\beta>0, f_{S}[S]$ in Eq. (13c) is minimized by

$$
S=0 .
$$

Substituting this into Eq. 13a), we find that $f_{g}\left[a_{1}, a_{2}, S=0\right]$ is minimized by

$$
g_{1}=g_{2}=\left\{\begin{array}{cc}
0, & \text { for } r \geq 0 \\
\sqrt{|r|}, & \text { for } r<0 .
\end{array}\right.
$$

which, as discussed above corresponds to the isotropiccrumpled and the isotropic-flat phase, respectively. This situation is illustrated in the $\beta-r$ plane in Fig. 1

For $\beta<0$, our strategy will be to determine the region of (meta-)stability for various phases. We first minimize $f_{g}\left[g_{1}, g_{2}, S\right]$, defined by Eq. 13a), for given $\alpha_{1}$ and $\alpha_{2}$, i.e. for given $S$ and $r$. It is clear that for fixed values of $\alpha_{1}$ and $\alpha_{2}$, a contour $f_{g}=$ Const. is an ellipse in the $\left(g_{1}, g_{2}\right)$ plane centered at $\left(\alpha_{1}, \alpha_{2}\right)$. Its principle axes are along two diagonal $(1, \pm 1)$ directions, as shown in Fig. 3 Depending on the values of $\alpha_{1}$ and $\alpha_{2}$, i.e. the center of the ellipse, the minimizer of $f_{g}\left[g_{1}, g_{2}, S\right]$ falls into three categories:

1. $\alpha_{1}>\alpha_{2}>0$ :

The ellipses are centered within the first quadrant. It is easy to see that $f_{g}$ is minimized by

$$
\left(g_{1}^{*}, g_{2}^{*}\right)=\left(\alpha_{1}, \alpha_{2}\right)=(S-r,-S-r),
$$

with a minimal value zero. This corresponds to a nematic-flat phase. Substituting these $g_{1}^{*}$ and $g_{2}^{*}$ into Eq. (12), we obtain:

$$
f=\frac{1}{2}\left(S^{2}+\beta\right)^{2},
$$

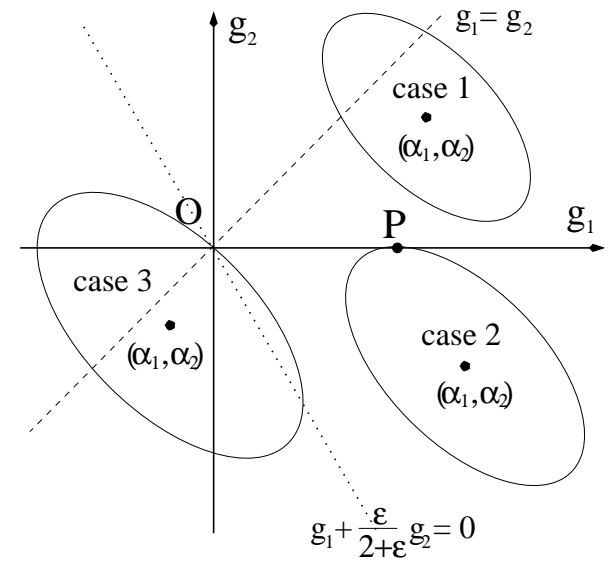

FIG. 3: Contour plot of $f_{g}=$ const., an ellipse centered at $\left(\alpha_{1}, \alpha_{2}\right)$ in the $\left(g_{1}, g_{2}\right)$ plane, where $\alpha_{1} \leq \alpha_{2}$, with principle axes along diagonal $(1,1)$ direction. As discussed in the text, in case $1, f_{g}$ is minimized by the center of the ellipse, that corresponds to a nematic-flat phase. In case 2 , it is minimized by the point $T$, where the ellipse is tangent with the positive $g_{1}$ axis. This corresponds to a nematic-tubule phase. In case 3 , $f_{g}$ is minimized by the origin $g_{1}=g_{2}=0$, which corresponds to a (nematic or isotropic) crumpled phase.

which, when minimized over $S$, gives the magnitude of nematic order parameter in the nematic-flat phase:

$$
S=\sqrt{|\beta|} .
$$

The condition of non-negativity of $g_{1,2}$ and $g_{2}^{*}=\alpha_{2}$, gives the condition of stability of the nematic-flat phase

$$
\begin{aligned}
g_{2}^{*} & =-(S+r) \\
& =-(r+\sqrt{|\beta|})>0,
\end{aligned}
$$

which leads to the phase boundary for the nematicflat phase illustrated in Fig. 1

2. $\alpha_{1}+\frac{\epsilon}{2+\epsilon} \alpha_{2}>0$ and $\alpha_{2}<0$ :

In this case, the centers of elliptic free energy contours are located outside the first quadrant, and therefore do not correspond to physical solution of the metric tensor, characterized by $g_{1,2} \geq 0$. Independent of $\alpha_{1}$,however, there is always one elliptic contour, which is tangent to the positive $g_{1}$ axis as illustrated in Fig. (3). It is straightforward to see that this tangent point, $\left(g_{1}^{*}>0, g_{2}^{*}=0\right)$ is the minimum of $f_{g}$ in the physical space of metrics, which corresponds to the nematic-tubule phase. At this tangent point, the partial derivative of $f_{g}$ with respect to $g_{1}$ vanishes, i.e.

$\left.\frac{\partial f_{g}}{\partial g_{1}}\right|_{\left(g_{1}^{*}, 0\right)}=2(2+\epsilon)\left(g_{1}^{*}-\alpha_{1}-\frac{\epsilon \alpha_{2}}{2+\epsilon}\right)=0$, 
which leads to

$$
\begin{aligned}
g_{1}^{*} & =\alpha_{1}+\frac{\epsilon \alpha_{2}}{2+\epsilon} \\
& =\frac{2(S-(1+\epsilon) r)}{2+\epsilon} .
\end{aligned}
$$

Substituting this $g_{1}^{*}$ and $g_{2}^{*}=0$ into Eq. (12), the rescaled total elastic free energy reduces to

$$
f=\frac{2(1+\epsilon)}{(2+\epsilon)}(S+r)^{2}+\frac{1}{2}\left(S^{2}+\beta\right)^{2} .
$$

The equilibrium value of $S$ is determined by minimizing this $f$ with constraints

$$
\begin{aligned}
\alpha_{2}<0 & \rightarrow S>-r, \\
\alpha_{1}+\frac{\epsilon \alpha_{2}}{2+\epsilon}>0 & \rightarrow S>(1+\epsilon) r .
\end{aligned}
$$

For a negative $r$, it is easy to see that as long as $r<\sqrt{|\beta|}$, there is always a positive value of $S$ that both minimizes Eq. (24) and satisfies constraints, Eqs. (25). The case of positive $r$ is more subtle. By a technically involved, but conceptually straightforward calculation, we find that for $\beta<-2-\frac{1}{2+\epsilon}$ (i.e. to the left of point $C_{1}$ in Fig. (1), a nematic tubule state is stable as long as $(1+\epsilon) r>\sqrt{|2+\beta|}$. For $-2(1+\epsilon) /(2+\epsilon)>\beta>-2-\frac{1}{2+\epsilon}$ (i.e. to the right of $C_{1}$, but to the left of $C_{2}$ in Fig. (1), we find that a metastable nematic-tubule state exists as long as

$$
\beta+\frac{2(1+\epsilon)}{2+\epsilon}<-3\left(\frac{(1+\epsilon) r}{2+\epsilon}\right)^{\frac{2}{3}} .
$$

And finally for $-2(1+\epsilon) /(2+\epsilon)<\beta<0$ (i.e. to the right of $C_{2}$ in Fig. 1), nematic tubule phase is stable if and only if $r<0$.

3. $\alpha_{1}+\frac{\epsilon}{2+\epsilon} \alpha_{2}<0$ and $\alpha_{2}<0$ :

In this case, there is no ellipse tangent with the positive $g_{1}$ axis, i.e. the nematic-tubule phase is not stable. There is however always one ellipse passing through the origin, $g_{1}^{*}=g_{2}^{*}=0$ (see Fig. 3) that minimizes $f_{g}$. Depending on the value of $S$, the corresponding phase may be nematic-crumpled or isotropic-crumpled. Setting $g_{1}=g_{2}=0$ in Eq. (12), we obtain

$f=\frac{1}{2} S^{4}+(2+\beta) S^{2}+\frac{1}{2} \beta^{2}+2(1+\epsilon) r^{2}$,

where we have used Eq. (14a) and Eq. (14b). Minimizing it over $S$, we find the equilibrium value of nematic order parameter

$$
S=\left\{\begin{array}{cl}
0, & \text { if } \beta+2>0 \\
\sqrt{|\beta+2|} & \text { if } \beta+2<0
\end{array}\right.
$$

Considering the self-consistent condition that defines case 3 , as well as the definition of $\alpha_{1}$ and $\alpha_{2}$, we find that for negative $\beta$, the isotropic-crumpled phase is stable if and only if

$$
\beta+2>0 \text { and } \quad r>0 .
$$

On the other hand the nematic-crumpled phase is stable if and only if

$$
\beta+2<0 \text { and } 0<\sqrt{|\beta+2|}<(1+\epsilon) r .
$$

We have thereby determined the region of metastability for all five phases admitted by the nematicelastomer membrane. These results are summarized in Fig. I1 In the $(\beta, r)$ plane the small triangular region between three critical points $C_{1}, C_{2}$ and $C_{3}$ is particularly interesting. This region is divided into two parts by the vertical line $\beta=-2$ passing through $C_{3}$. In the left part, both nematic-tubule and nematic-crumpled phases are metastable. In the right part, both nematic-tubule and isotropic-crumpled phase are metastable. Consequently, there exists a first-order transition line between $C_{1}$ and $C_{2}$. This line is illustrated schematically by a dashed curve in Fig. 1. It connects a second-order nematictubule - to - nematic-crumpled transition boundary on the left to a second-order nematic-tubule - to - isotropiccrumpled phase boundary on the right. The exact position of this first-order line, which can in principle be determined from comparing free energy of different phases, is not essential to our discussion. Outside the triangular region, all phase transitions are continuous in our meanfield analysis.

For completeness, in Table. II we list the coordinates of the three critical points, $C_{1}, C_{2}$ and $C_{3}$ in the $(\beta, r)$ plane. It is interesting to note that distances between any two of these points, and therefore the triangular area, vanishes as $\epsilon$ becomes large, corresponding to an incompressible limit of vanishing shear-to-bulk moduli ratio. Due to the same reason, in the limit of small shear modulus, the discontinuity of first-order transition line (jump of order parameter or other physical quantities) vanishes.

\begin{tabular}{|l|l|l|l|}
\hline \hline & $C_{1}$ & $C_{2}$ & $C_{3}$ \\
\hline$\beta$ & $-2-\frac{1}{2+\epsilon}$ & $-2+\frac{2}{2+\epsilon}$ & -2 \\
\hline$r$ & $\frac{1}{(1+\epsilon) \sqrt{2+\epsilon}}$ & 0 & 0 \\
\hline \hline
\end{tabular}

TABLE II: Coordinates of three critical points $C_{1}, C_{2}$ and $C_{3}$.

\section{PHANTOM NEMATIC-TUBULE PHASE}

Among all phases that we have identified in the preceeding section, the nematic tubule phase is the most interesting one. As we have just found, it is characterized by a nonzero nematic order and a metric tensor with one positive eigenvalue, i.e.

$$
\underline{\underline{Q}}_{0}=\left(\begin{array}{cc}
S & 0 \\
0 & -S
\end{array}\right), \quad \underline{\underline{g}}_{0}=\left(\begin{array}{cc}
g_{1} & 0 \\
0 & 0
\end{array}\right) .
$$


Even though the ground state configuration of this nematic tubule phase is identical to that of the tubule phase studied in Ref. 10], as we will show in this section, its elasticity and thermal fluctuations differ qualitatively, due to the fact that rotational symmetry is spontaneously broken, both in the reference space and in the target space.

\section{A. Harmonic Fluctuations}

We first study harmonic thermal fluctuations in the nematic tubule phase. Without loss of generality we pick the intrinsic (reference space) membrane axes such that in the ground state the $x$-axis coincide with the extended direction of tubule, with tubule therefore crumpled along the $y$-axis. Similarly, our choice of the target space coordinate system orients the tubule along $X$-axis of the embedding space. With this choice, the tubule groundstate conformation is given by

$$
\mathbf{R}_{0}(\mathbf{r})=\zeta x \hat{X},
$$

where $g_{1}=\zeta^{2}$ can be calculated by minimizing the total free energy Eq. (9).

To study thermal fluctuations about the nematictubule ground state, we follow the parameterization introduced in Ref. 10] for the study of an intrinsicallyanisotropic tubule. A deviation away from the ground state can be parameterized by a one-dimensional (scalar) phonon field $u(\mathbf{r})$ and a two-dimension undulation field $\mathbf{h}(\mathbf{x})$, defined by

$$
\mathbf{R}(\mathbf{r})=(\zeta x+u(\mathbf{r})) \hat{X}+\mathbf{h}(\mathbf{r}) .
$$

The corresponding metric tensor, Eq. (2), is then given by

$$
\begin{aligned}
g_{x x}^{0} & =\left(\zeta+\partial_{x} u\right)^{2}+\left(\partial_{x} \mathbf{h}\right)^{2} \\
g_{x y}^{0} & =\left(\zeta+\partial_{x} u\right)\left(\partial_{y} u\right)+\left(\partial_{x} \mathbf{h}\right) \cdot\left(\partial_{y} \mathbf{h}\right), \\
g_{y y}^{0} & =\left(\partial_{y} u\right)^{2}+\left(\partial_{y} \mathbf{h}\right)^{2} .
\end{aligned}
$$

The Lagrange strain tensor is defined as half the deviation of the metric tensor from its ground state value $\underline{\underline{g}}$, i.e.

$$
u_{a b}=\frac{1}{2}\left(g_{a b}-g_{a b}^{0}\right) .
$$

Its components are given by

$$
\begin{aligned}
& u_{x x}=\zeta\left(\partial_{x} u\right)+\frac{1}{2}\left(\partial_{x} u\right)^{2}+\frac{1}{2}\left(\partial_{x} \mathbf{h}\right)^{2}, \\
& u_{x y}=\frac{1}{2}\left(\zeta+\partial_{x} u\right)\left(\partial_{y} u\right)+\frac{1}{2}\left(\partial_{x} \mathbf{h}\right) \cdot\left(\partial_{y} \mathbf{h}\right), \\
& u_{y y}=\frac{1}{2}\left(\partial_{y} u\right)^{2}+\frac{1}{2}\left(\partial_{y} \mathbf{h}\right)^{2} .
\end{aligned}
$$

Fluctuations of the nematic order parameter $\underline{Q}$ about its ground-state value $\underline{\underline{Q}}_{0}$ can be parameterized by two variables $\tau$ and $\sigma$ defined by:

$$
\underline{\underline{Q}}=\underline{\underline{Q}}+\delta \underline{\underline{Q}}=\left(\begin{array}{cc}
S+\tau & \sigma \\
\sigma & -S-\tau
\end{array}\right)
$$

They correspond to longitudinal and transverse excitations of the nematic order, respectively. Their physical significance can be readily seen by comparing Eq. (37) with the more usual parameterization of nematic fluctuation in terms of rotation angle $\theta$ and magnitude fluctuation $\delta S$ :

$$
\underline{\underline{Q}}=(S+\delta S)\left(\begin{array}{cc}
\cos 2 \theta & \sin 2 \theta \\
\sin 2 \theta & -\cos 2 \theta
\end{array}\right) .
$$

We find that up to linear order of $\delta S$ and $\theta$,

$$
\sigma \approx 2 S \theta, \quad \tau \approx \delta S .
$$

Therefore the longitudinal component $\tau$ describes fluctuations in the magnitude of nematic anisotropy about $S$, while the transverse component $\sigma$ is proportional to the rotation of the nematic director.

When expressed in terms of these scalar fields and the Lagrange strain tensor, the nematic-tubule free energy, Eq. (9), becomes

$$
\begin{aligned}
f \underline{\underline{g}, \underline{Q}]} & =\frac{(1+\epsilon)}{2}\left(2 r+\zeta^{2}+2 u_{x x}+2 u_{y y}\right)^{2} \\
& +\frac{1}{2}\left(\beta+2 \sigma^{2}+2(S+\tau)^{2}\right)^{2}+2\left(\sigma-2 u_{x y}\right)^{2} \\
& +2\left(S-\zeta^{2} / 2+\tau-u_{x x}+u_{y y}\right)^{2}
\end{aligned}
$$

The condition that $\underline{\underline{g}}$ and $\underline{\underline{Q}} 0$, as given by Eq. (31), minimize the elastic free energy density $f[\underline{\underline{g}}, \underline{\underline{Q}}]$ guarantees that in Eq. (40), terms that are linear in $\tau$ or $u_{x x}$ strictly vanish, and thereby leads to two equations that determine the ground-state order parameters $S$ and $\zeta$ :

$$
\begin{array}{r}
-2 S+2 r(1+\epsilon)+(2+\epsilon) \zeta^{2}=0, \\
4 S^{3}+2 S(1+\beta)-\zeta^{2}=0 .
\end{array}
$$

Finally, eliminating the strain components $u_{a b}$ in favor of the phonon $(u)$ and height undulation (h) fields using Eqs. (36), and keeping only upto quadratic terms, we obtain the harmonic free energy density of the nematictubule:

$$
\begin{aligned}
f[\sigma, \tau, u, \mathbf{h}] & =f[\underline{\underline{g}}, \underline{\underline{Q}}]-f\left[\underline{\underline{g}}_{0}, \underline{\underline{Q}}_{0}\right] \\
& =K_{\sigma}(\nabla \sigma)^{2}+C_{\sigma}\left(\sigma-\alpha_{\sigma} \partial_{y} u\right)^{2} \\
& +K_{\tau}(\nabla \tau)^{2}+C_{\tau}\left(\tau-\alpha_{\tau} \partial_{x} u\right)^{2} \\
& +B_{u}\left(\partial_{x} u\right)^{2}+K_{u}\left(\partial_{y}{ }^{2} u\right)^{2} \\
& +B_{h}\left(\partial_{y} \mathbf{h}\right)^{2}+K_{h}\left(\partial_{x}{ }^{2} \mathbf{h}\right)^{2}
\end{aligned}
$$


where

$$
\begin{aligned}
C_{\sigma} & =2\left(1+2 S^{2}+\beta\right), \\
C_{\tau} & =2\left(1+6 S^{2}+\beta\right), \\
\alpha_{\sigma} & =\frac{\zeta}{1+2 S^{2}+\beta}, \\
\alpha_{\tau} & =\frac{\zeta}{1+6 S^{2}+\beta}, \\
B_{u} & =2(2+\epsilon) \zeta^{2}, \\
B_{h} & =4 S-2 \zeta^{2} .
\end{aligned}
$$

There are several salient features of the nematic-tubule free energy density, Eq. (42), that are worth pointing out. Firstly, terms that are first-derivative of $\mathbf{h}$ with respect to the ordered direction, $x$, namely $\left(\partial_{x} \mathbf{h}\right)^{2}$, do not appear in $f$. Small $\partial_{x} \mathbf{h}$ corresponds to an infinitesimal rotation in the embedding space of the tubule's extension axis, that we have arbitrarily chosen to point along $\hat{X}$ and therefore must not cost any elastic free energy. Therefore absence of the $\left(\partial_{x} \mathbf{h}\right)^{2}$ term is a direct consequence of the underlying rotational invariance of the embedding space, that a tubule, extended along $\hat{X}$, spontaneously breaks. 10 Secondly, $f$ exhibits a linear coupling of the longitudinal fluctuation of the nematic-order parameter, $\tau$ to the longitudinal strain, $\partial_{x} u$. This corresponds to the linear coupling between $\underline{Q}$ and the metric tensor $\underline{g}$ in the original model Eq. (9) and encodes the fact that $\stackrel{\underline{\underline{Q}}}{\bar{n}}$ matic order induces uniaxial strain and vice versa. Lastly and most importantly, the transverse fluctuation of the nematic order, $\sigma$ is "minimally" 32 coupled to the transverse strain component $\partial_{y} u$, with the coefficient of the linear coupling $\sigma \partial_{y} u$ precisely such that it forms a complete square with two other terms $\sigma^{2}$ and $\left(\partial_{y} u\right)^{2}$. This ensures that a small and nonzero transverse strain $\partial_{y} u$ (corresponding to a reorientation of the uniaxial strain axis) can be completely compensated by a uniform perturbation $\sigma$, which corresponds to a global rotation of the nematic director within the reference space, so that the overall free energy change is exactly zero. This property is a consequence of the underlying rotational invariance of the membrane's reference space, spontaneously broken by the nematic order, and is therefore expected on general symmetry principles. One consequence of such coupling is that integration over the nematic director field, $\sigma$, leads to an effective elastic theory for the nematic-tubule, in which the shear term $\left(\partial_{y} u\right)^{2}$ is absent. The resulting extra "softness" is the essential feature that qualitatively distinguishes a nematic-tubule studied here from a tubule in an explicitly anisotropic polymerized membrane studied by Radzihovsky and Toner. 10 It reflects the fact that in a nematic-tubule the rotational symmetry in the reference space is only spontaneously broken, and therefore a simultaneous rotation of the nematic order and the metric tensor in the reference space does not cost energy. For mechanical stability we have therefore added to the free energy Eq. (42) two curvature terms, $K_{u}$ and $K_{h}$, that would otherwise be unimportant for smooth defor- mations.

To study thermal undulations of the nematic-tubule it is convenient to integrate out fluctuations of nematic order parameter, $\tau$ and $\sigma$, in a direct analogy of integrating out the nematic director to study properties of the smectic phase. This leads to an effective elastic Hamiltonian for the nematic-tubule (in harmonic approximation)

$$
\begin{aligned}
H_{\mathrm{eff}}[u, \mathbf{h}] & =\frac{1}{2} \int d x d y\left[B_{u}\left(\partial_{x} u\right)^{2}+K_{u}\left(\partial_{y}{ }^{2} u\right)^{2}\right. \\
& \left.+B_{h}\left(\partial_{y} \mathbf{h}\right)^{2}+K_{h}\left(\partial_{x}{ }^{2} \mathbf{h}\right)^{2}\right], \\
& =\frac{1}{2} \int d q_{x} d q_{y}\left[\left(B_{u} q_{x}^{2}+K_{u} q_{y}^{4}\right)|u(\mathbf{q})|^{2}\right. \\
& \left.+\left(B_{h} q_{y}^{2}+K_{h} q_{x}^{4}\right)|\mathbf{h}(\mathbf{q})|^{2}\right],
\end{aligned}
$$

where in the last equation we have also expressed the elastic Hamiltonian in terms of the Fourier components defined by:

$$
\begin{aligned}
u(\mathbf{q}) & =\int d x d y e^{-i \mathbf{q} \cdot \mathbf{r}} u(\mathbf{r}), \\
\mathbf{h}(\mathbf{q}) & =\int d x d y e^{-i \mathbf{q} \cdot \mathbf{r}} \mathbf{h}(\mathbf{r}) .
\end{aligned}
$$

From $H_{\text {eff }}$ we observe that at the harmonic level, fluctuations of $\mathbf{h}$ field and the phonon field $u$ are decoupled from each other. Furthermore, the in-plane phonon-field $u$ elasticity is identical to that of extensively studied twodimensional smectic liquid crystal with layers extended along $y$. 19, 33] Therefore we can immediately adopt known results for a two-dimensional smectic to fluctuations of the nematic-tubule phase. 19, 33. For example, for a membrane with intrinsic size $L_{x} \times L_{y}$, the bulk contribution to mean-squared fluctuations (see below for contribution from other modes) for $u$ is given by:

$$
\begin{aligned}
\left\langle u^{2}\right\rangle & =\frac{1}{B_{u}} \sqrt{\frac{L_{x}}{2 \pi a_{u}}} \psi_{u}\left(2 \pi a_{u} L_{x} / L_{y}^{2}\right) \\
& = \begin{cases}\frac{1}{\sqrt{2} \pi B_{u}} \sqrt{\frac{L_{x}}{2 \pi a_{u}}}, & \text { if } L_{y}^{2} \gg 2 \pi a_{u} L_{x} ; \\
\frac{1}{(2 \pi)^{2}} \frac{1}{B_{u}} \frac{L_{y}}{a_{u}}, & \text { if } L_{y}^{2} \ll 2 \pi a_{u} L_{x},\end{cases}
\end{aligned}
$$

Therefore, thermal fluctuations of the phonon field, $u$ grow without bound with membrane's intrinsic size $\left(L_{x}\right.$, $\left.L_{y}\right)$. This growth of thermal fluctuations of the $u$ field is a signature of the qualitative importance of the hitherto neglected nonlinear elastic terms.

From Eq. (42) we observe that at long length scales (beyond $\sqrt{K_{\sigma} / C_{\sigma}}$ ) the nematic orientation field, $\sigma$ is locked by the "minimal coupling" term to transverse strain fluctuations, $\partial_{y} u$. This, together with dimensional analysis allows us to relate the asymptotic scaling of the nematic director field, $\sigma$ to that of the phonon field $u$ through

$$
\left\langle\sigma^{2}\right\rangle \propto \frac{1}{L_{y}^{2}}\left\langle u^{2}\right\rangle
$$


Despite a strong growth of $\left\langle u^{2}\right\rangle$ appearing in Eq. (46), we therefore find that the in-plane nematic orientational fluctuations remains finite in the thermodynamic limit.

From $H_{\text {eff }}$ we observe that the undulation field, $\mathbf{h}$, is also governed by the same two-dimensional smectic anisotropic elasticity, except that the roles of $x$ and $y$ axes are interchanged. At the harmonic level, it is identical to that of a permanently anisotropic membrane studied in Ref. 10. As discussed in great detail there, two related quantities can be defined to describe the tubule geometry at a finite temperature, tubule radius of gyration, $R_{G}$ (thickness) and tubule roughness, $h_{r m s}$ as follows:

$$
\begin{aligned}
R_{G}^{2} & =\left\langle\left|\mathbf{h}(x, 0)-\mathbf{h}\left(x, L_{y}\right)\right|^{2}\right\rangle, \\
h_{r m s}^{2} & =\left\langle\left|\mathbf{h}(0, y)-\mathbf{h}\left(L_{x}, y\right)\right|^{2}\right\rangle .
\end{aligned}
$$

As indicated in Fig $2 R_{G}$ measures the radius of a typical cross-section of the tubule that is perpendicular to the nematic order and tubule axis, while $h_{r m s}$ measures wandering of the tubule transverse its backbone. As discussed by Radzihovsky and Toner [10] fluctuations of these two quantities are strongly affected by the so-called zero modes, that correspond to Fourier modes of the undulation field $\mathbf{h}$ with one vanishing wave vector component. Adapting these results to our case, we find:

$$
\begin{aligned}
R_{G}\left(L_{x}, L_{y}\right) & =L_{y}^{1 / 4} S_{R}\left(L_{x} / \sqrt{L_{y}}\right), \\
h_{r m s}\left(L_{x}, L_{y}\right) & =L_{x}^{1 / 2} S_{h}\left(L_{x} / \sqrt{L_{y}}\right),
\end{aligned}
$$

where two crossover functions $S_{R}$ and $S_{h}$ satisfy

$$
\begin{aligned}
& S_{R}(x) \rightarrow \begin{cases}\frac{1}{\sqrt{x}} & x \rightarrow 0, \\
\text { constant } & x \rightarrow \infty ;\end{cases} \\
& S_{h}(x) \rightarrow \begin{cases}\text { constant } & x \rightarrow 0, \\
x & x \rightarrow \infty .\end{cases}
\end{aligned}
$$

For an approximately square size membrane $\left(L_{x} \simeq L_{x} \simeq\right.$ $L$ ), we find that $h_{r m s}$ scales linearly with $L$, while $R_{G} \propto$ $L^{1 / 4}$. Finally, we note that quantities similar to Eq. (48) can also be defined for the fluctuations of the phonon field $u$. Their asymptotic forms are also given by Eq. (49), with the role of $x$ and $y$ axes interchanged.

As was found for explicitly-anisotropic tubules [10], we expect that inclusion of the so-far ignored self-avoidance and elastic nonlinearity (both from in-plane phonon as well as height undulation) will swell the nematic-tubule and further stabilize it against thermal fluctuations. In order to perform such a study, we will need a formalism of elastic free energy for the nematic tubule that includes all relevant nonlinearities. We derive this nonlinear elasticity in the next subsection and leave the study of it to a future research.

\section{B. Nonlinear theory for nematic-tubule}

Our goal now is to derive the fully rotationallyinvariant (in both the reference and target spaces) free energy describing nematic-tubule. This turns out to be simpler within the purely elastic formulation, in which, as discussed in Sec III the in-plane nematic orientational degrees of freedom have been formally integrated out. The analysis here, then parallels our work on nonlinear elasticity of three-dimensional, bulk nematic elastomers. 17]

In such purely elastic approach nematic-tubule's free energy is a functional of the deviation of metric tensor $\underline{\underline{g}}$ from its equilibrium values $\underline{\underline{g_{0}}}$. Since the elastic free energy density should be invariant under arbitrary rotations in both the reference and the target spaces, for homogeneous deformations and in two dimensions it must be a function of only two scalar functions of the metric tensor [34]:

$$
\begin{aligned}
& S_{1}=\operatorname{Tr} \underline{\underline{g}}, \\
& S_{2}=\operatorname{Tr} \underline{\underline{g}}^{2} .
\end{aligned}
$$

In the nematic-tubule phase, we can expand the effective elastic free energy density around the ground state corresponding to the metric tensor $\underline{\underline{g_{0}}}$, Eq. (31):

$$
\begin{aligned}
f_{\mathrm{eff}}\left[S_{1}, S_{2}\right] & =f_{\mathrm{eff}}\left[S_{1}^{0}, S_{2}^{0}\right]+A_{1} \delta S_{1}+A_{2} \delta S_{2} \\
& +\frac{1}{2} B_{1} \delta S_{1}^{2}+B_{12} \delta S_{1} \delta S_{2}+\frac{1}{2} B_{2} \delta S_{2}^{2} \\
& +O\left(\delta S_{i}^{3}\right),
\end{aligned}
$$

where

$$
\begin{aligned}
& \delta S_{1}=\operatorname{Tr} \underline{\underline{g}}-\operatorname{Tr} \underline{\underline{g}}=2 \operatorname{Tr} \underline{\underline{u}}, \\
& \delta S_{2}=\operatorname{Tr} \underline{\underline{g}}^{2}-\operatorname{Tr} \underline{\underline{g}}_{0}^{2}=4 \operatorname{Tr} \underline{\underline{g_{0}}} \underline{\underline{u}}+4 \operatorname{Tr} \underline{\underline{u}}^{2} .
\end{aligned}
$$

As can be checked a posteriori, terms of order $\delta S_{i}^{3}$ and higher are irrelevant at long-scales in the renormalization-group sense and can therefore be omitted, as we have done above.

Using Eq. (54), we may further express the elastic free energy density in terms of the Lagrange strain tensor. To proceed, however, we note the following relations between $\delta S_{1}$ and $\delta S_{2}$ :

$$
\begin{aligned}
\delta S_{1} \delta S_{2} & =2 \zeta^{2} \delta S_{1}^{2}+\text { irrelevant } \\
& =8 \zeta^{2}(\operatorname{Tr} \underline{\underline{u}})^{2}+\text { irrelevant } \\
\delta S_{2}^{2} & =4 \zeta^{4} \delta S_{1}^{2}+\text { irrelevant } \\
& =16 \zeta^{4}(\operatorname{Tr} \underline{\underline{u}})^{2}+\text { irrelevant. }
\end{aligned}
$$

Substituting these relations, together with Eqs. (54) into Eq. (53), we obtain the following nonlinear elastic free energy density for the nematic tubule:

$$
\begin{aligned}
f[\underline{\underline{u}}] & =f_{\mathrm{eff}}\left[S_{1}, S_{2}\right]-f_{\mathrm{eff}}\left[S_{1}^{0}, S_{2}^{0}\right] \\
& =2\left(A_{1}+2 \zeta^{2} A_{2}\right) \operatorname{Tr} \underline{\underline{u}}+4 A_{2}\left(-\zeta^{2} u_{y y}+\operatorname{Tr} \underline{\underline{u}}^{2}\right) \\
& +2\left(B_{1}+4 \zeta^{2} B_{12}+4 \zeta^{4} B_{2}\right)(\operatorname{Tr} \underline{\underline{u}})^{2} .
\end{aligned}
$$

Using the fact that $\underline{\underline{g}}=0$, Eq. (31) is a true ground state, together with the observation, from Eqs. (36), that Tru 
and $u_{y y}$ are, respectively, linear and quadratic in the displacement fields, $u$ and $\mathbf{h}$, we find that the coefficient of $\operatorname{Tr} \underline{\underline{u}}$ identically vanishes, namely

$$
A_{1}+2 \zeta^{2} A_{2}=0 .
$$

To express the elastic free energy in terms of the phonon field $u$ and the undulation field $\mathbf{h}$, we furthermore note the following two identities:

$$
\begin{aligned}
\operatorname{Tr} \underline{\underline{u}} & =\zeta \partial_{x} u+\frac{1}{2}(\nabla u)^{2}+\frac{1}{2}(\nabla \mathbf{h})^{2}, \\
-\zeta^{2} u_{y y}+\operatorname{Tr}_{\underline{\underline{u}}}^{2} & =\left(\zeta \partial_{x} u+\frac{1}{2}(\nabla u)^{2}+\frac{1}{2}(\nabla \mathbf{h})^{2}\right)^{2} \\
& -\frac{1}{2}\left(\zeta \partial_{y} \mathbf{h}+\left(\partial_{x} u\right)\left(\partial_{y} \mathbf{h}\right)-\left(\partial_{y} u\right)\left(\partial_{x} \mathbf{h}\right)\right)^{2} \\
& + \text { irrelevant }
\end{aligned}
$$

Finally, in order to further simplify the notation, we rescale the coordinates in the reference space according to:

$$
x \rightarrow \zeta^{-1} x,
$$

thereby obtaining our final expression for the nonlinear elastic free energy density of a nematic tubule:

$$
\begin{aligned}
f[u, \mathbf{h}] & =\frac{1}{2} B_{u}\left[\left(\partial_{x} u\right)+\frac{1}{2}(\nabla u)^{2}+\frac{1}{2}(\nabla \mathbf{h})^{2}\right]^{2} \\
& +\frac{1}{2} B_{h}\left[\partial_{y} \mathbf{h}\left(1+\partial_{x} u\right)-\left(\partial_{y} u\right)\left(\partial_{x} \mathbf{h}\right)\right]^{2} \\
& +\frac{1}{2} K_{u}\left(\partial_{y}{ }^{2} u\right)^{2}+\frac{1}{2} K_{h}\left(\partial_{x}{ }^{2} \mathbf{h}\right)^{2},
\end{aligned}
$$

with the effective bulk elastic moduli given by:

$$
\begin{aligned}
& B_{u}=8 \zeta^{4} A_{2}+4 \zeta^{4}\left(B_{1}+4 \zeta^{2} B_{12}+4 \zeta^{4} B_{2}\right), \\
& B_{h}=-4 \zeta^{4} A_{2} .
\end{aligned}
$$

Despite our ignoring of a number of (at long-scales) subdominant terms, it is easy to verify that our final expression for the elastic free energy density, Eq. (60), is actually exactly invariant under arbitrary rotations in both the reference and the target spaces. To see this we first note that tubule's reference ground-state conformation, using the rescaled coordinate system Eq.(159), is given by

$$
\mathbf{R}_{0}(\mathbf{x})=\hat{X} x .
$$

Under arbitrary rotation both in the reference space and in the target space, it becomes

$$
\mathbf{R}(\mathbf{x})=\hat{N}_{0}\left(\hat{n}_{0} \cdot \mathbf{x}\right) \equiv \hat{X}\left(x+u_{0}(\mathbf{x})\right)+\mathbf{h}_{0}(\mathbf{x}),
$$

characterized by target and reference-space unit vectors, $\hat{N}_{0}$ and $\hat{n}_{0}$, respectively. This situation is illustrated in Fig. (IVB). Without loss of generality, we may parameterize these two unit vectors as

$$
\begin{aligned}
\hat{N}_{0} & =\hat{X} \cos \phi+\hat{Y} \sin \phi, \\
\hat{n}_{0} & =\hat{x} \cos \theta+\hat{y} \sin \theta .
\end{aligned}
$$
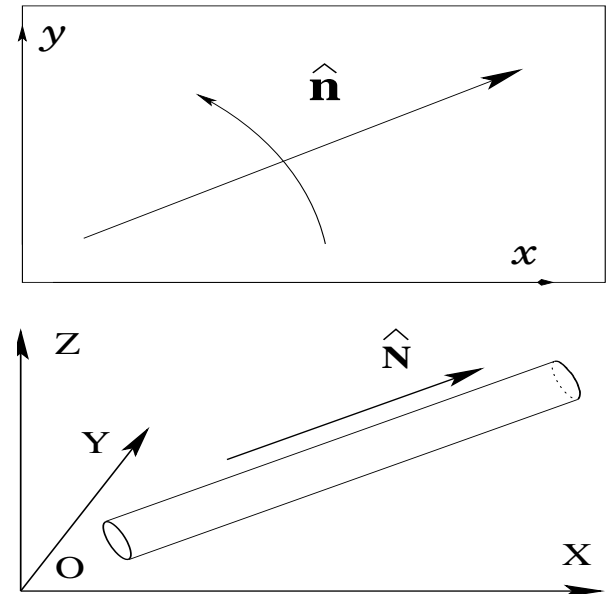

FIG. 4: A ground state conformation of a nematic tubule can be parameterized by two unit vectors $\hat{n}_{0}$ and $\hat{N}_{0}$, Eq. [63), that respectively describe the orientation of the tubule in the reference and target spaces. In the reference state Eq. (62), $\hat{n}_{0}$ and $\hat{N}_{0}$ are chosen to be $\hat{x}$ and $\hat{X}$ respectively.

From Eq. (63), it is easy to see that relative to the reference state $\mathbf{R}_{0}$, Eq.(62), this pure rotation corresponds to a phonon field $u_{0}(\mathbf{x})$ and an undulation field $\mathbf{h}_{0}(\mathbf{x})$ given by

$$
\begin{aligned}
u_{0}(\mathbf{x}) & =\zeta x(\cos \theta \cos \phi-1)+\zeta y \sin \theta \cos \phi \\
\mathbf{h}_{0}(\mathbf{x}) & =\zeta \hat{Y} \sin \phi(x \cos \theta+y \sin \theta) .
\end{aligned}
$$

Substituting these two equations into $f[u, \mathbf{h}], \mathrm{Eq} 60$ we find, that, indeed, as demanded by rotational invariance, the nonlinear elastic free energy strictly vanishes for an arbitrary rotation in both the reference and target spaces.

\section{CONCLUSION}

In this paper, we have presented a Landau theory for polymerized membrane with spontaneous in-plane nematic order. We have analyzed the mean-field phase diagram and studied harmonic thermal fluctuations in the nematic-tubule phase. We have also derived a nonlinear elastic free energy for the nematic-tubule, which will be an essential starting point for future analysis of elastic nonlinearities and self-avoiding interaction that we have so far ignored. Such a study is necessary in order to understand the physics of any realistic membrane exhibiting spontaneous nematic tubule phase.

\section{Acknowledgments}

We thank T. C. Lubensky for discussion and acknowledge support by the NSF MRSEC DMR-0213918 (LR), 
DMR-0321848 (LR), David and Lucile Packard Foundation (LR), as well as NSF DMR02-05858 (XX)and DOE DEFG02-91ER45439(XX). We also thank Harvard De- partment of Physics and KITP at UC Santa Barbara, where part of this work was done, for hospitality.
[1] Also referred as fixed connectivity, tethered, or crystal membrane in various references.

[2] For a review, see Statistical Mechanics of Membranes and Interfaces, edited by D. R. Nelson, T. Piran, and S. Weinberg (World Scientific, Singapore, 1989).

[3] D. R. Nelson and L. Peliti, J. Phys. (Paris) 48, 1085(1987).

[4] J. A. Aronovitz and T. C. Lubensky, Phys. Rev. Lett. 60, 2634(1988); J. A. Aronovitz, L. Golubovic, and T. C. Lubensky, J. Phys.(Paris) 50, 609(1989).

[5] P. LeDoussal and L. Radzihovsky, Phys. Rev. Lett. 69, 1209(1992).

[6] Y. Kantor and D. R. Nelson, Phys. Rev. Lett. 58, 2774(1987).

[7] M. Paczuski, M. Kardar, and D. R. Nelson, Phys. Rev. Lett. 60, 2638(1988).

[8] E. Guitter, F. David, S. Leibler, and L. Peliti, Phys. Rev. Lett. 61, 2949(1988).

[9] L. Radzihovsky, to appear in the 2nd Edition of the Statistical Mechanics of Membranes and Surface, edited by D. R. Nelson, T. Piran, and S. Weinberg, World Scientific, Singapore.

[10] L. Radzihovsky and J. Toner, Phys. Rev. Lett. 75, 4752(1995); Phys. Rev. E 57, 1832 (1998).

[11] M. Bowick, M. Falcioni, and G. Thorleifsson, Phys. Rev. Lett. 79, 885(1997); cond-mat/9705059

[12] L. Golubovic and T. C. Lubensky, Phys. Rev. Lett. 63, 1082(1989).

[13] M. Warner and E. M. Terentjev, Prog. Polym. Sci. 21, 853(1996), and the reference therein.

[14] E. M. Terenjev, J. Phys. Cond. Mat. 11, R239(1999).

[15] H. Finkelmann, I. Kundler, E.M. Terentjev, and M. Warner, J. Phys. II 7, 1059(1997); G.C. Verwey, M. Warner, and E.M. Terentjev, J. Phys. II (France) 6, 1273-1290(1996); M. Warner, J. Mech. Phys. solids 47, 1355(1999).

[16] T. C. Lubensky, R. Mukhopadhyay, L. Radzihovsky, and X. Xing, Phys. Rev. E 66, 011702(2002); cond-mat/0112095

[17] X. Xing and L. Radzihovsky, Europhys. Lett. 61, 769 (2003); Phys. Rev. Lett. 90, 168301(2003).

[18] O. Stenull and T. C. Lubensky, Europhys. Lett. 61, 776 (2003); Phys. Rev. E 69, 021807(2004).

[19] X. Xing, R. Mukhopadhyay, T. C. Lubensky, and L. Radzihovsky, Phys. Rev. E 68, 021108 (2003).

[20] Similar phenomena take place in other "soft" anisotropic systems, such as smectic 25] and columnar liquid crystal phases 25], vortex lattices in putative magnetic superconductors [26], and bulk nematic elastomers [16, 17, 18].

[21] Stability of an isotropic-flat phase at finite temperature is ensured by length scale dependent bending modulus that is infinitely enhanced by thermal fluctuations. Therefore this finding of a finite, length-scale independent bendingrigidity modulus for flat nematic elastomer membranes suggests that this nematic-flat phase might indeed be unstable to thermal fluctuations. However, we have also shown that for $D<3$, new in-plane elastic nonlinearities which is not included in our analysis near $D=4$, become qualitatively important, and can very well lead to the sufficient enhancement of the bending rigidity sufficient to stabilize a nematic-flat phase of a $2 \mathrm{D}$ membrane. At the moment, this question remains unresolved.

[22] R. Podgornik, Phys. Rev. E 52, 5170(1995).

[23] However, topological defects in these two systems are qualitatively different.

[24] As the word "phantom" suggests, we will completely ignore the effects of self-avoiding interaction. It should be made clear that the reason we ignore self-avoidance in this work is not its unimportance. On the contrary, past research experience in this field shows that except for the flat phase, self-avoiding interaction is qualitatively important to the long length scale properties of polymerized membranes. The primary object of this paper is to study the global phase diagram of a liquid-crystalline tethered membrane. We believe that self-avoidance does not destroy, but rather modifies, various phases that we will identify in this paper. Therefore we will leave the technically involved study of self-avoiding interaction to a separate publication.

[25] L. Radzihovsky and J. Toner, Phys. Rev. B 60 206(1999); Phys. Rev. Lett. 78 4414(1997).

[26] L. Radzihovsky, A. M. Ettouhami, K. Saunders, and J. Toner, Phys. Rev. Lett. 87, 027001 (2001).

[27] L. Radzihovsky and D. R. Nelson, Phys. Rev. A 44, 3525(1991), Europhys. Lett. 16, 79(1991), Phys. Rev. A , 46 7474(1992); D. C. Morse, T. C. Lubensky and G. S. Grest, Phys. Rev. A 45, R2151(1992); D. C. Morse, T. C. Lubensky, Phys. Rev. A 46, 1751(1992).

[28] L. S. Hirst and C. R. Safinya, unpublished.

[29] Such an intrinsic nematic order parameter $\underline{Q}$ may, for example, characterize a spontaneous lipid tilt-order or alignment of liquid crystal polymers making up a nematic elastomer membrane. It should be intuitively clear that the component of such orientational order normal to the membrane, should have no anisotropic effects on the elasticity of the tethered membrane. Consequently $\underline{\underline{Q}}$ the nematic order parameter is a well-defined reference-space rank-two tensor that can couple to membrane's metric tensor $\underline{g}$. This contrasts qualitatively with distinct physical realization of, for example, a tethered membrane fluctuating inside a nematic liquid crystal. In this latter case the appropriate nematic order parameter is a tensor in the embedding space that can only couple to tangent vectors of a membrane.

[30] P G. de Gennes and J. Prost, The Physics of Liquid Crystals, Clarendon Press, Oxford, 1993.

[31] This crumpling transition is second order at a mean field level and is supported by numerical simulation. RG analysis, however, shows that it may be discontinuous.

[32] This is mathematically identical to what's called a "minimal" coupling of a charged-field and the corresponding 
gauge field in gauge theories that ensures gauge invariance, with the identification of $\sigma$ with the gauge field, $u$ the phase of the charged field and $1 / \alpha_{\sigma}$ the charge.

[33] T. Salditt, I. Koltover, J. O. Rädler, and C. R. Safinya, Phys. Rev. E 58, 889(1998).
[34] This is because in two dimensions the metric tensor $g$ is a $2 \times 2$ matrix and has only two rotationally-invariant independent degrees of freedom, i.e. two eigenvalues. 